\title{
Endometrioma Presented as Acute Abdomen in a Peritoneal Dialysis Patient: A Case Report and Literature Review
}

\author{
Zatul Akmar Ahmad (D) \\ Fathi Ramly (D) ${ }^{\prime}$ \\ Akmal Zulayla Mohd Zahid' \\ Bahiyah Abdullah (D) ${ }^{1,2}$ \\ Muhammad lqbal Abdul $\mathrm{Hafidz}^{3}$ \\ 'Department of Obstetrics and \\ Gynaecology, Faculty of Medicine, \\ Universiti Teknologi MARA, Sungai Buloh \\ Campus, Selangor, Malaysia; ${ }^{2}$ Maternofetal \\ and Embryo (MatE) Research Group, \\ Faculty of Medicine, Universiti Teknologi \\ MARA, Sungai Buloh Campus, Selangor, \\ Malaysia; ${ }^{3}$ Department of Medicine, \\ Faculty of Medicine, Universiti Teknologi \\ MARA, Sungai Buloh Campus, Selangor, \\ Malaysia
}

\begin{abstract}
Laparoscopic management in patients on peritoneal dialysis offers good postoperative outcome and early resumption to peritoneal dialysis. This is a report of a 43-yearold woman with end-stage kidney disease on peritoneal dialysis presented with left iliac fossa pain due to a large endometrioma. Emergency diagnostic laparoscopy was performed with careful laparoscopic entry, Tenckhoff catheter care and anti-adhesive application. The patient underwent temporary hemodialysis for two weeks before full resumption to peritoneal dialysis. She was planned for GnRH analogue for three months post-operatively. In conclusion, laparoscopy is a safe and advantageous route of surgery for ovarian endometrioma in patients on peritoneal dialysis. However, mindful surgical strategies are needed to ensure the Tenckhoff catheter's longevity and early resumption to peritoneal dialysis postoperatively.
\end{abstract}

Keywords: laparoscopy, Tenckhoff, endometrioma, renal replacement therapy

\section{Introduction}

Endometriosis is a chronic inflammatory disorder defined by viable, estrogensensitive endometrial-like glands and stroma outside the uterus. ${ }^{1}$ About $10-15 \%$ of reproductive-aged women are reported to suffer from pelvic endometriosis, the most typical benign gynaecological pathology among reproductive-aged women. ${ }^{2}$ Despite its prevalence and many studies looking into its pathophysiology, to date, this disease remains poorly understood. There are various hypothetical explanations proposed to explain the condition and its varied clinical presentations. ${ }^{1,2}$ It has been established that the severity of the disease does not correlate with symptoms that the patient may have. ${ }^{2}$

Peritoneal dialysis (PD) treatment is widely available and is well accepted for endstage kidney disease in Malaysia. PD makes up $10.1 \%$ of renal replacement therapy (RRT) in the country. An essential aspect of managing PD is maintaining the treatment longevity, and this is done mainly by preventing PD infections. PD longevity is also a crucial factor to consider in the case of abdominal surgery. Open abdominal surgery will almost always mean that it will undoubtedly result in the conversion of PD to hemodialysis, either temporarily or permanently. Therefore, more recent literature recommends the use of the laparoscopic approach in various pathological conditions. However, there remain multiple aspects of concern about the surgical technique itself during the laparoscopic procedures and the subsequent postoperative care.
Correspondence: Bahiyah Abdullah Department of Obstetrics and Gynaecology Faculty of Medicine, Universiti Teknologi MARA, Sungai Buloh Campus, Jalan Hospital, Selangor, 47000, Malaysia

$\mathrm{Tel}+603-6126500$

Fax +60 3-61265224

Email bahiyah@uitm.edu.my 
The burden of chronic kidney disease (CKD) worldwide is estimated to be at $9.1 \%{ }^{3}$ The prevalence of CKD amongst adults in Malaysia is estimated at $15.48 \%{ }^{4}$ This is due to the high prevalence of diabetes, hypertension and obesity amongst Malaysian adults based on the four yearly performed National Health and Morbidity Survey. ${ }^{5}$ This has led to many patients diagnosed with end-stage kidney disease (ESKD), and the prevalence shows an increasing trend every year. Malaysia has one of the highest numbers of ESKD affecting the younger age groups, and females make up $46 \%$ of the total population of ESKD patients in the country. ${ }^{6}$ Therefore, more women within the reproductive age group with CKD may present with a gynaecological problem.

Nevertheless, the prevalence of PD patients with endometriosis is scarce. Several literatures have described that severe endometriosis leads to ureteric obstruction and renal impairment. There have been no previously reported cases of an endometrioma in a PD patient to the best of our knowledge. Therefore, we report a rare case of laparoscopic management of an endometrioma in a PD patient and her subsequent postoperative care.

Special considerations should be taken into account when managing these women, both pharmacologically and surgically. The management plan requires both aspects of the diseases to be addressed in terms of therapeutic objective, side effects of treatment, and fertility implications.

\section{Case Summary}

A 43-year-old, a single nulliparous with multiple medical comorbidities, presented with a seven-day history of worsening left pelvic pain. It started as a sudden sharp pain at left pelvic and iliac fossa radiated to lower back with pain score oscillating between 3 to 6 out of 10 . There was no aggravating factor, but it was partially relieved by potent analgesia. She denied any urinary tract infection symptoms, nor any change in her bowel habits.

She had underlying end-stage kidney disease (ESKD) due to diabetic nephropathy and was on peritoneal dialysis. She was also anemic due to her ESKD and was on erythropoietin treatment. She was recently started on Irbesartan for uncontrolled hypertension and was on fluid restriction of $500 \mathrm{~mL} /$ day due to decompensated heart failure. She attained menarche at 12 years old with a regular cycle every three months. Due to her chronic medical illness, her menses became scanty with a cycle length of up to 6 months. However, she denied any dysmenorrhea, abnormal vaginal discharge or intermenstrual bleeding episode.
Physical examination upon presentation showed normal vital signs and her BMI was $38 \mathrm{~kg} / \mathrm{m} 2$. The abdomen was tender at left iliac fossa, with no rebound tenderness or guarding. The Tenckhoff catheter was in situ with normal surrounding skin.

Ultrasound scan showed uterus axial measuring $6.3 \mathrm{~cm}$ $\mathrm{x} 3.5 \mathrm{~cm}$ with normal endometrial thickness. A complex left adnexal mass measured $10 \mathrm{~cm} \times 10 \mathrm{~cm}$ in diameter, with mixed solid and cystic echogenic content with no other ultrasound features suggestive of malignancy. CT scan of abdomen and pelvis reported the presence of a leftsided cystic mass $8.3 \times 8.4 \times 8.9 \mathrm{~cm}$ arising from left adnexa (Figure 1). There was fine intralesional septation at the inferior and superior aspect of the mass. The mass displaced the normal anteverted uterus to the right. Radiological feature of peritoneal fluid was not evident in both ultrasound and CT scan. This suggested that a ruptured cyst was less likely. Tumor markers were normal (CA-125 11U/mL, AFP 2.8ng/mL, CEA 1.8 ng/mL).

The patient continued to have persistent left iliac fossa pain despite potent analgesia. Therefore, an emergency diagnostic laparoscopy was performed for suspected twisted ovarian cyst. Before the procedure, we carefully wrapped the Tenckhoff catheter with a clean gauze. The scar from her previous Tenckhoff catheter insertion was close to the umbilicus, raising a concern about potential adhesions in the surrounding area. Thus, we decided to create pneumoperitoneum via the primary port insertion at Palmer's point. After peritoneal survey through the primary port, auxiliary ports were inserted at Le Huang point, right and left iliac fossa (Figure 2).

Intraoperatively, there were no intraperitoneal adhesions noted in the abdomen or pelvis. There was a large

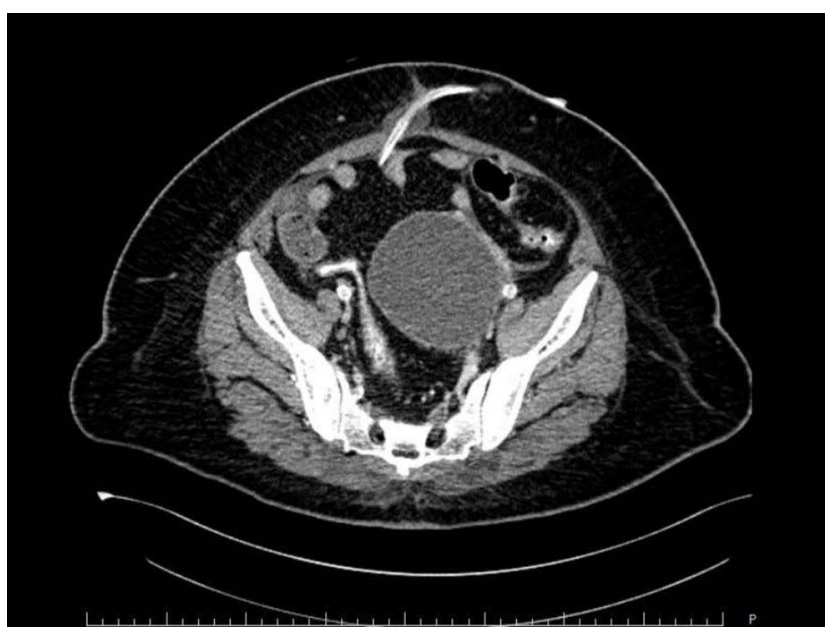

Figure I CT scan of the pelvis findings. 


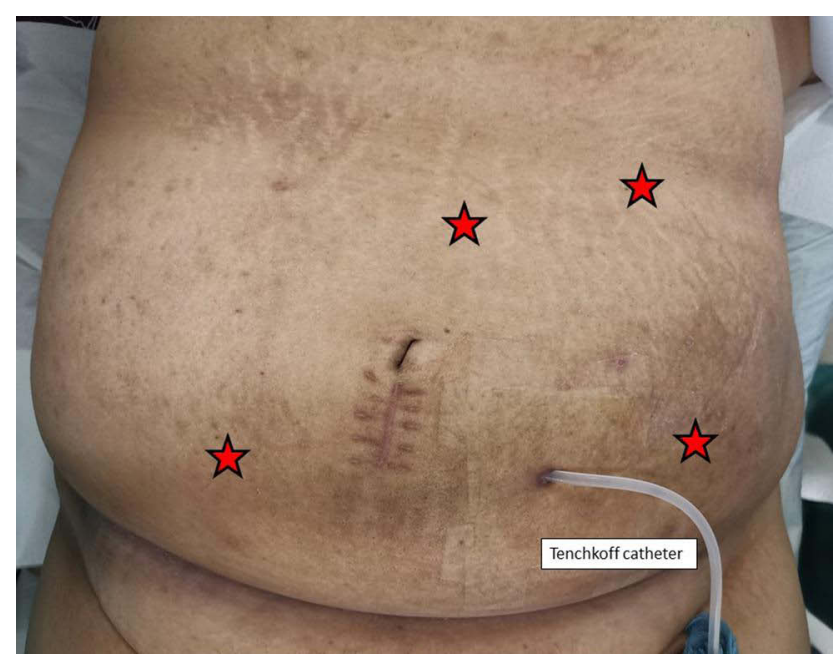

Figure 2 Ports placement.

left ovarian cyst measuring $10 \times 10 \mathrm{~cm}$ with no evidence of rupture or torsion (Figure 3). The cyst was adherent to the sigmoid colon posteriorly. The peritoneal tip of the Tenckhoff catheter was at the vesicouterine pouch, away from the cyst. The cyst ruptured during manipulation releasing thick chocolate fluid material which was consistent with an endometrioma. Adhesiolysis and left ovarian cystectomy was performed, and ovarian reconstruction was performed using Vicryl 2/0 suture. The Tenckhoff catheter was flushed with normal saline to remove the blood clot and to maintain patency. Anti-adhesive agent $\left(\right.$ Protescal $^{\mathrm{TM}}$ ) was applied to the surgical area.

She had an uncomplicated recovery post-operatively. The histopathological examination result of the cyst wall confirmed an endometrioma. The patient then underwent temporary hemodialysis (HD) for two weeks before recommencing her PD using the same Tenckhoff catheter.

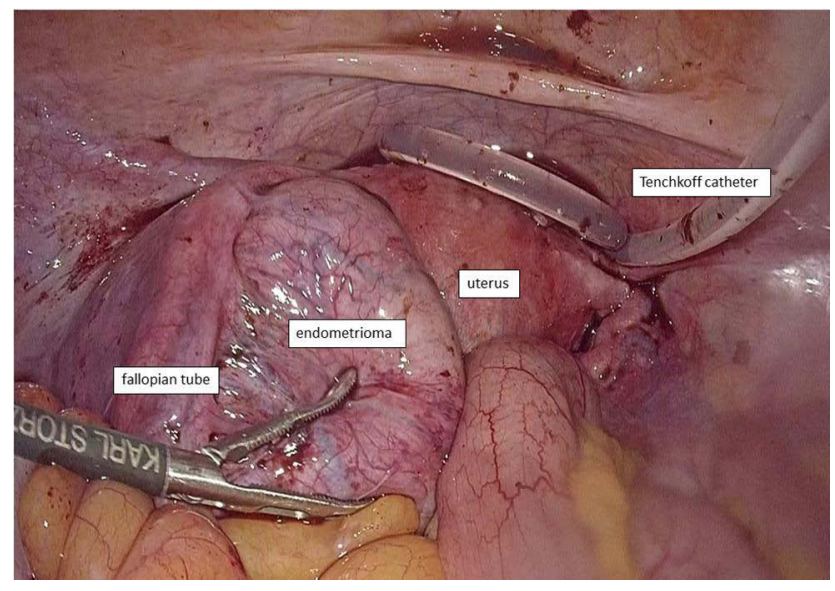

Figure 3 Intraoperative finding.
This interval period was to allow proper healing of the surgical wound, especially the port sites. The Tenckhoff catheter was flushed weekly during this period. She was planned for GnRH analogue for three months postoperatively.

\section{Discussion}

A few aspects of this patient's management plan require deliberation due to lack of evidence on renal failure patients' care on regular PD with endometrioma. This patient had infrequent menses with longer cycles since she had CKD, likely due to oligo-ovulation or anovulation that is common among CKD patients, given uremia that causes hypothalamic-pituitary-gonadal dysfunction. Another potential cause of anovulation is raised serum leptin. It has been found that serum leptin levels are generally significantly elevated in patients with kidney failure compared with age- and body mass index-matched controls. ${ }^{7,8}$ Patients with endometriosis may be asymptomatic or symptomatic with dysmenorrhea, dyspareunia, subfertility and chronic pelvic pain. However, this patient did not have dysmenorrhea, and being a single lady, dyspareunia and subfertility are not an issue.

Diagnosis can be made clinically in a particular patient who presented with typical clinical history. However, in this case, history and examination alone were not enough to achieve a definitive diagnosis. An ultrasound is a helpful investigation tool in making a diagnosis. The ultrasound features that may suggest an endometrioma include unilocular cyst (most commonly), ground-glass appearance, and no vascularization on color Doppler. ${ }^{9}$ With the advance of technology and in expert hands, ultrasound can also demonstrate findings of deep infiltrating endometriosis. In this case, we saw the less frequent endometrioma features with septation and solid cystic appearance. Considering the rarity of an endometrioma presence in PD patients, and that patients on chronic dialysis treatment have an increased risk of cancer, with the kidney and bladder being the most common sites, ${ }^{10}$ the ovarian tumor should be suspected. Despite no reported association with increased risk of ovarian malignancy in a dialysis patient, we performed CT scan given the atypical ultrasound features on top of lack of typical presenting symptoms of endometriosis in this patient.

Patients presenting with an acute abdomen due to ovarian cyst accidents require emergency surgery. However, for patients on PD treatment, the surgery route involves considering multiple factors including potential 
pathology, patient's hemodynamic status and underlying comorbidities. There is a high incidence of perioperative complications, such as dialysate fluid leakage, wound dehiscence, incisional hernia, peritonitis or hemoperitoneum in traditional laparotomy surgery in PD patients. ${ }^{11}$ Abdominal surgery comprising large abdominal incision may frequently require removal of the catheter or at least temporary interruption of peritoneal dialysis to prevent dialysate leakage. ${ }^{12}$ Performing an open abdominal surgery in a patient on PD may also lead to the development of intra-abdominal adhesions and loss of peritoneal membrane integrity, leading to an inability to continue PD modality effectively. ${ }^{13}$ Therefore, a laparoscopic approach is more advantageous as it involves minimal breaching of the abdominal wall, reduced abdominal adhesion, and a decreased risk for postoperative leakage of dialysate. Therefore, PD can be resumed in the postoperative period with less chance of fluid leakage or peritonitis than open abdominal surgery. ${ }^{14-16}$

Besides, there are other known benefits of laparoscopic surgery, such as faster recovery, less postoperative pain, shorter hospital stays and improved cosmesis, which makes laparoscopic surgery the gold standard for the management of this case. Furthermore, this patient had ESKD on PD. She also had poorly controlled type 2 diabetes mellitus and obesity; therefore, a laparoscopic surgery involving smaller wound incision will decrease her risk for wound infection and other complications such as wound breakdown. The addition of perioperative antibiotics also will lower her risk of peritonitis.

There are multiple case reports on the laparoscopic approach in various abdominal surgeries, including hemicolectomy, ${ }^{15,17}$ hysterectomy, ${ }^{18}$ cholecystectomy, ${ }^{14,19}$ bariatric surgery ${ }^{20}$ and nephrectomy. ${ }^{11,21} \mathrm{Up}$ to writing this report, there is no reported laparoscopic cystectomy performed in a patient with peritoneal dialysis. This is based on our literature search on PubMed, Google Scholar, LISTA, Web of Science and the Cochrane Library from 1990 using the keywords "laparoscopy", "laparoscopic", "cystectomy" and "peritoneal dialysis". A review of literature by Mari et al in 2017 concluded that laparoscopy had been successfully applied to several abdominal surgical procedures in patients on PD with good outcomes and early PD resumption. ${ }^{16}$

Currently, there is no consensus on the recommended time for PD resumption. The reported time for PD resumption ranged from 1 day up to 4 weeks postoperatively, depending on the type of surgery. ${ }^{12,16,18,22}$
During PD, there will be increased intra-abdominal pressure with peritoneal dialysate infusion. Thus, postoperative leakage of dialysate through the wounds is a concern. Kakuda et al in 2017 reported inability to resume PD following total laparoscopic hysterectomy for early endometrial cancer due to dialysate leakage. They speculated that dislocation of the Tenckhoff catheter to the upper abdomen might have contributed to the PD leakage. ${ }^{18}$ Due to this concern, we opted for a temporary transition to HD before PD's resumption. Fortunately, our patient managed to resume her PD modality three weeks following the surgery without any complications. Several publications have recently demonstrated the immediate postoperative continuation of $\mathrm{PD}$ in laparoscopic gastric banding or bypass surgery. ${ }^{13}$ PD recommencement can be achieved with a strict PD regimen of initiating low volume PD on day one after surgery. Gradual dialysate volume increment over the next few weeks is practiced until toleration of full volume of PD. ${ }^{13}$ This is undoubtedly an option considered for similar cases in the future.

Initially, many cases reported laparoscopy being performed with removal of the peritoneal catheter. Recently, more case reports document that laparoscopic surgery is feasible without prior peritoneal catheter removal. ${ }^{15}$ However, more reports on the safety of performing laparoscopic surgery without Tenckhoff catheter removal remains required. ${ }^{17}$ There are a few considerations that should be taken into account during the laparoscopic surgery with peritoneal catheter in-situ. First, the Tenckhoff catheter should be cleaned and covered with sterile gauze/ plastic cover. Second, the laparoscopic ports should not be inserted too close to the Tenckhoff catheter site to avoid accidentally hitting the peritoneal catheter. In this case, the first port was inserted at Palmer's point. Then all the auxiliary ports were inserted under direct vision. The port placement is crucial to avoid injury upon the entry of port sites. We were careful to minimize handling or contact of the Tenckhoff catheter to avoid its dislocation, which may contribute to PD leakage problems. ${ }^{11}$ Third, we took care to reduce the incidence of Tenckhoff catheter blockage. After we ensured hemostasis, the peritoneal cavity was irrigated to avoid clot formation in the peritoneal cavity, potentially causing blockage of the Tenckhoff catheter. Flushing the Tenckhoff catheter is another strategy to prevent Tenckhoff catheter dysfunction such as slow fill or drain and obstruction that had been proposed. ${ }^{23,24}$ Therefore, we also flushed the catheter 
with normal saline before the end of surgery and weekly post-operatively. Another aspect of concern is the development of postoperative adhesions that may cause peritoneal catheter dysfunction. However, the nature of laparoscopy being associated with less risk of forming adhesions is another advantage. ${ }^{12}$

This case and other reported cases further support that laparoscopic surgery is a safe and effective alternative route of surgery for several abdominal surgical procedures, including cystectomy in patients on PD with good outcomes and early resumption of PD.

The redevelopment or recurrence of endometrioma is a significant concern post-operatively. Commencement of pharmacological intervention would generally be considered to minimize disease progression.

GnRH analogues are an effective therapy for endometriosis; however, their use is limited by hypoestrogenism's associated symptoms, particularly in long-term treatment, where it is associated with decreased bone mineral density. Another option of therapy is dienogest (DNG), a fourthgeneration selective progestin. DNG with specific binding to progesterone receptors has a local effect on endometriotic lesions, with less androgenic, estrogenic, glucocorticoid or mineralocorticoid activity, and minimal impact on the metabolic parameters. ${ }^{25}$ Prescription of $2 \mathrm{mg}$ /day is effective in treating endometriosis and the results obtained are similar to those with GnRH analogues with good efficacy and tolerability. ${ }^{26}$ Unfortunately, DNG is contraindicated in diabetic patients with vascular involvement, making this not suitable for this case.

To date, there is a lack of literature on long-term management of endometriosis in an ESKD patient. We counselled her for GnRH analogue treatment for three months post-surgery to reduce the risk of recurrence. However, considering that she has amenorrhea with likely anovulatory cycles, the recurrence chances are probably very low. Therefore, expectant management with surveillance of disease progression without any pharmacological intervention should be another acceptable option.

\section{Conclusion}

The management of endometrioma in a renal failure patient requiring peritoneal dialysis requires careful combined management between the gynecologist and nephrologist. A minimally invasive surgical approach is safe and should be the gold standard management when feasible. Special consideration should be given upon choosing the pharmacological and surgical technique. Like the general population, laparoscopy is a safe and more advantageous route of surgery for ovarian endometrioma either electively or in an emergency setting in a patient on peritoneal dialysis. Careful counselling about the pros and cons of hormonal treatment to prevent recurrence are essential.

\section{Acknowledgments}

Written informed consent for publication of the clinical details and images was obtained from the patient of this case report.

\section{Disclosure}

All the authors report no conflicts of interest in this work.

\section{References}

1. Falcone T, Flyckt R. Clinical management of endometriosis. Obstet Gynecol. 2018;131(3):557-571. doi:10.1097/AOG.00000000000 02469

2. Mehedintu C, Plotogea MN, Ionescu S, Antonovici M. Endometriosis still a challenge. J Med Life. 2014;7(3):349-357.

3. Bikbov B, Purcell CA, Levey AS; GBD Chronic Kidney Disease Collaboration. Global, regional, and national burden of chronic kidney disease, 1990-2017: a systematic analysis for the Global Burden of Disease Study 2017. Lancet. 2020;395(10225):709-733. doi:10.1016/S0140-6736(20)30045-3

4. Saminathan TA, Hooi LS, Mohd Yusoff MF, et al. Prevalence of chronic kidney disease and its associated factors in Malaysia; findings from a nationwide population-based cross-sectional study. $B M C$ Nephrol. 2020;21(1):344. doi:10.1186/s12882-020-01966-8

5. Institute for Public Health. National Health and Morbidity Survey (NHMS) 2019: non-communicable diseases, healthcare demand, and health literacy_Key Findings; 2020. Available from: http://iptk.moh. gov.my/images/technical_report/2020/4_Infographic_Booklet_ NHMS_2019_-_English.pdf. Accessed June 15, 2021.

6. National Renal Registry. 26th report of the Malaysian Dialysis and Transplant Registry; 2018. Available from: https://www.msn.org.my/ nrr/mdtr2018.jsp. Accessed June 15, 2021.

7. Ghazizadeh S, Lesanpezeshki M. Reproduction in women with end-stage renal disease and effect of kidney transplantation. Iran J Kidney Dis. 2007;1(1):12-15.

8. Wolf G, Chen S, Han DC, Ziyadeh FN. Leptin and renal disease. Am J Kidney Dis. 2002;39(1):1-11. doi:10.1053/ajkd.2002.29865

9. Moro F, Leombroni M, Testa AC. Ultrasound imaging in endometriosis. Obstet Gynecol Clin North Am. 2019;46(4):643-659. doi:10.1016/j.ogc.2019.07.004

10. Maisonneuve P, Agodoa L, Gellert R, et al. Cancer in patients on dialysis for end-stage renal disease: an international collaborative study. Lancet. 1999;354(9173):93-99. doi:10.1016/S0140-6736(99) 06154-1

11. Rais-Bahrami S, Romero FR, Lima GC, Kohanim S, Kavoussi LR. Reinstatement of continuous ambulatory peritoneal dialysis after transperitoneal laparoscopic nephrectomy. Urology. 2006;68 (4):715-717. doi:10.1016/j.urology.2006.04.032

12. Mutter D, Marichal J-F, Heibel F, Marescaux J, Hannedouche T. Laparoscopy: an alternative to surgery in patients treated with continuous ambulatory peritoneal dialysis. Nephron. 1994;68 (3):334-337. doi:10.1159/000188396

13. Nguyen AH, Naljayan M, Yazdi F, Reisin E. Laparoscopic sleeve gastrectomy in a patient on peritoneal dialysis. Kidney Int Rep. 2020;5(12):2361-2364. doi:10.1016/j.ekir.2020.09.014 
14. Magnuson TH, Bender JS, Campbell KA, Ratner LE. Cholecystectomy in the peritoneal dialysis patient. Unique advantages to the laparoscopic approach. Surg Endosc. 1995;9 (8):908-909. doi:10.1007/BF00768890

15. Auricchio S, Mari G, Galassi A, et al. Laparoscopic left hemicolectomy for colon cancer in peritoneal dialysis patients: a valid and safe surgical technique to ensure peritoneal dialysis survival. Perit Dial Int. 2016;36(6):695-699. doi:10.3747/pdi.2015.00259

16. Mari G, Scanziani R, Auricchio S, Crippa J, Maggioni D. Laparoscopic surgery in patients on peritoneal dialysis: a Review of the Literature. Surg Innov. 2017;24(4):397-401. doi:10.1177/ 1553350617708723

17. Attard JA, Attard A. Laparoscopic right hemicolectomy in an automated peritoneal dialysis patient without removal of the PD catheter: a Case Report. Case Rep Surg. 2014;2014:492567. doi:10.1155/2014/ 492567

18. Kakuda M, Kobayashi E, Tanaka Y, Ueda Y, Yoshino K, Kimura T. Total laparoscopic hysterectomy for endometrial cancer in a renal transplantation patient receiving peritoneal dialysis: case report and literature review. J Obstet Gynaecol Res. 2017;43(7):1232-1237. doi:10.1111/jog. 13337

19. Ha JF, Chandraratna H. Laparoscopic cholecystectomy in chronic ambulatory peritoneal dialysis. Ochsner J. 2009;9(1):17-19.
20. Valle GA, Kissane BE, de la Cruz-muñoz N. Successful laparoscopic bariatric surgery in peritoneal dialysis patients without interruption of their CKD6 treatment modality. Adv Perit Dial. 2012;28:134-139.

21. Malavade TS, Bargman JM. The outcome of nephrectomy in peritoneal dialysis patients. Adv Perit Dial. 2013;29:25-28.

22. Bellizzi V, Giannoulias D, Al-Sunaid M, et al. Gynecological surgery: not a contraindication for continuation of CAPD. Perit Dial Int. 2003;23(2):193-196. doi:10.1177/089686080302300217

23. Wesseling KM, Pierik RG, Offerman JJ. Laparoscopic therapy in a CAPD patient with perforated appendicitis without removal of the peritoneal catheter. Nephrol Dial Transplant. 2003;18(9):1929-1930. doi:10.1093/ndt/gfg289

24. Okada H, Ryuzaki M, Kotaki S, et al. Thoracoscopic surgery and pleurodesis for pleuroperitoneal communication in patients on continuous ambulatory peritoneal dialysis. Am J Kidney Dis. 1999;34 (1):170-172. doi:10.1016/S0272-6386(99)70126-5

25. Köhler G, Faustmann TA, Gerlinger C, Seitz C, Mueck AO. A dose-ranging study to determine the efficacy and safety of 1,2 , and $4 \mathrm{mg}$ of dienogest daily for endometriosis. Int $J$ Gynaecol Obstet. 2010;108(1):21-25. doi:10.1016/j.ijgo.2009.08.020

26. Andres Mde P, Lopes LA, Baracat EC, Podgaec S. Dienogest in the treatment of endometriosis: systematic review. Arch Gynecol Obstet. 2015;292(3):523-529. doi:10.1007/s00404-015-3681-6

\section{Publish your work in this journal}

The International Journal of Women's Health is an international, peerreviewed open-access journal publishing original research, reports, editorials, reviews and commentaries on all aspects of women's healthcare including gynecology, obstetrics, and breast cancer. The manuscript management system is completely online and includes a very quick and fair peer-review system, which is all easy to use. Visit http://www.dovepress.com/testimonials.php to read real quotes from published authors. 